\title{
THE DIVISION OF SPACE AND THE POISSON DISTRIBUTION
}

\section{RICHARD COWAN, ${ }^{*}$ CSIRO Division of Mathematics and Statistics, Lindfield}

This letter reports on a fascinating new result concerning the Poisson distribution. The result arose during a study of cellular division within epithelia (fundamental 'sheet-like' tissues of the body) and has general relevance to the partitioning to two-dimensional space whenever the compartments of space are successively cleaved or fractured. A more complete account of the biological context from which the mathematical problem is abstracted is given in Cowan and Morris (1988) but, in this note, we focus on part of that work which (in a surprising way) involves the Poisson distribution.

Consider a convex polygon with $k$ sides. We propose to divide it with a straight line joining two of the $k$ sides. Suppose that the choice of sides is stochastic with the simplest probability measure, namely, each of the ${ }^{k} C_{2}$ choices being equally likely. Having chosen which two sides are to be joined, it is unimportant where on these sides the line's endpoints are positioned, except that neither should be placed at a vertex of the polygon.

After the dividing line is placed we have two convex polygons, the 'daughters' of the original 'mother cell'. Next we independently apply the same stochastic rules to each daughter polygon, simultaneously dividing them both and so creating four convex polygons. Division proceeds in this way.

Let $X_{n}$ be the number of sides of a randomly chosen polygon from generation $n$ (the initial $k$-sided polygon is in generation 0). As $n$ tends to infinity, it turns out that $X_{n}-3$ becomes Poisson distributed with mean 1 for any $k$.

To show this, let $Y_{n}(r)$ be the number of polygons with $r$ sides in generation $n$. Given $Y_{n}(r)$, the chance that a randomly sampled member of generation $n$ has $r$ sides is $Y_{n}(r) / 2^{n}$. Unconditionally, this chance is $E Y_{n}(r) / 2^{n}$. Let $\boldsymbol{m}_{n}$ be a row vector whose elements are $E Y_{n}(r)$, $r=3,4, \cdots$. This initial vector $\boldsymbol{m}_{0}$ contains mainly zeros but with 1 in the appropriate position to indicate the starting polygon. It is easy to show that

$$
\boldsymbol{m}_{n}=\boldsymbol{m}_{n-1} \boldsymbol{M}=\cdots=\boldsymbol{m}_{0} M^{n},
$$

where

$$
\boldsymbol{M}=\left(\begin{array}{cccccc}
1 & 1 & & & & \\
\frac{2}{3} & \frac{2}{3} & \frac{2}{3} & & & \\
\frac{1}{2} & \frac{1}{2} & \frac{1}{2} & \frac{1}{2} & & \\
\frac{2}{5} & \frac{2}{5} & \frac{2}{5} & \frac{2}{5} & \frac{2}{5} & \\
\frac{1}{3} & \frac{1}{3} & \frac{1}{3} & \frac{1}{3} & \frac{1}{3} & \frac{1}{3} \\
. & . & . & . & . & .
\end{array}\right) .
$$

The elements of $\boldsymbol{M}$ can be indexed by $(i, j)$, but here it is convenient to let both $i$ and $j$ run over $3,4, \cdots$, whereupon the $(i, j)$ element is the mean number of $j$-sided 'daughter' polygons which an $i$-sided mother can produce. This $(i, j)$ th element is $2 /(i-1)$ for $j=3,4, \cdots, i+1$, zero otherwise. Define the row vector $\boldsymbol{p}_{n}$ as $\boldsymbol{m}_{n} / 2^{n}$; thus the elements of $\boldsymbol{p}_{n}$ are the chances of sampling an $r$-sided polygon in generation $n(r=3,4, \cdots)$. From (1),

$$
\boldsymbol{p}_{n}=\boldsymbol{p}_{n-1} \boldsymbol{M} / 2=\cdots=\boldsymbol{p}_{0}(\boldsymbol{M} / 2)^{n} .
$$

We are interested in the convergence of $\boldsymbol{p}_{n}$, and thus of $(M / 2)^{n}$. If a limit exists then it

Received 23 September 1988; revision received 5 December 1988.

${ }^{*}$ Present address: CSIRO Division of Biotechnology, Box 184, North Ryde, NSW 2113, Australia. 
provides the limiting distribution of $X_{n}-3$. To think of this in a related setting, consider the Markov chain that arises when, after each polygon is split, one of the two daughters is randomly selected and discarded whilst the other is retained and further divided. The number of sides for successive retained polygons is an irreducible Markov chain with stochastic transition matrix $\boldsymbol{M} / 2$. Issues of convergence are as for our original problem.

If the equation $\boldsymbol{p}=\boldsymbol{p M} / 2$ has a solution $\boldsymbol{p}$ whose elements sum to 1 , then this establishes that the chain is ergodic, that the chain's state vector $\boldsymbol{p}_{n}$ converges and that $\boldsymbol{p}$ is the limit (Cox and Miller (1965), \$3.8). If we write $\boldsymbol{p}$ as $\left(p_{3}, p_{4}, \cdots\right)$, it is a simple matter to show that $p_{r}=e^{-1} /(r-3)$ !. (First show $p_{3}=p_{4}$ and $\left.p_{r}=p_{r-1}-p_{r-2} /(r-3), r>4\right)$. In the original problem where no cells are discarded, the proof of convergence of $\boldsymbol{p}_{n}$ to this $\boldsymbol{p}$ follows an identical argument. In that context $p_{r}$ is the limit of $E Y_{r}(n) / 2^{n}$ as $n$ tends to $\infty$. Thus the limiting Poisson result is established.

This interesting link between the division of space and the Poisson distribution is not easy to rationalise using analogies with other problems where the Poisson distribution finds application. It appears to be a completely new use of that remarkable distribution (though the remarks in the companion letter of Mecke deal with a seemingly unrelated stochastic process where the same matrix $M / 2$ arises).

It can easily be shown that the result applies also to the situation where, instead of simultaneous division of all cells in the same generation, any individual cell divides in the interval $(t, t+d t)$ with probability $\lambda d t+o\left(d t^{2}\right)$. A randomly sampled polygon at some large time $t$ will have $X_{t}$ sides, with $X_{t}-3$ distributed as Poisson.

\section{References}

Cowan, R. AND Morris, V. B. (1988) Division rules for polygonal cells. J. theor. Biol. 131, 33-42.

Cox, D. R. AND Miller, H. D. (1965) The Theory of Stochastic Processes. Methuen, London.

Mecke, J. (1989) A note on Cowan's process of space division. Adv. Appl. Prob. 21, 235-236. 